\title{
Improvement in the laser system for the A0 TTF Photoinjector
}

\author{
Xi Yang
}

March 10, 2003

\begin{abstract}
The production of high charge and high brightness electron beams places increasingly challenging demands on the drive laser used at the A0 photoinjector in the Fermilab. The IR and UV laser pulse lengths need to be optimized for such purpose. We have experimentally investigated two different ways to change the UV laser pulse length on the cathode; either by changing the bandwidth of the oscillator or by changing the distance between two compression gratings, the UV laser pulse length can be varied in the range of $3 \mathrm{ps}$ to $30 \mathrm{ps}$. Also the strong correlation between the UV laser energy and the IR laser pulse length has been studied, and the result is applied to achieve the UV laser energy of $18 \mu \mathrm{J} /$ pulse.
\end{abstract}

\section{Laser System}

The FNAL/TTF RF photoinjector has been designed to match the requirements of the TTF accelerator with high bunch charge $(10 \mathrm{nC})$, low emittance $(<20 \mathrm{~mm}-\mathrm{mrad})$ and high duty cycle $(1 \%)$. A solid-state laser system to meet these requirements has been developed at the Fermilab.[1] The laser produces a $1 \mathrm{MHz}$ train of up to 800 equalamplitude pulses with up to $1 \mathrm{~mJ}$ per pulse at the wavelength of $1054 \mathrm{~nm}$. The laser pulse train is produced by a phase-stabilized, mode-locked, Nd:YLF oscillator and a fast selection Pockels cell. The pulses are amplified in a Nd:glass amplifier chain consisting of a multipass rod ampifier and a 2-pass rod amplifier. The laser system uses Chirped Pulse Amplification (CPA) to produce 10ps pulses. After the fourth harmonic generation of the laser to $263.5 \mathrm{~nm}, 18 \mu \mathrm{J}$ per UV laser pulse can be obtained.

A schematic diagram of the laser system is shown in Figure 1. A mode-locked $\mathrm{Nd}$ :YLF oscillator produces a low energy, continuous pulse train at $81.25 \mathrm{MHz}$. Pulses from the oscillator are stretched and chirped in a $2 \mathrm{~km}$ fiber. After the fiber, a train of 800 pulses is selected at $1 \mathrm{MHz}$ from the oscillator pulse train by a fast, low voltage, lithium 
tantalite (LTA) pockels cell. Each of the 800 pulses is injected into a multipass amplifier which contains a flashlamp-pumped, $1 / 4 \times 6$ inch Nd:glass rod amplifier and a fast $\mathrm{KD} * \mathrm{P}$ Q-switch pockels cell. Each pulse is trapped in the cavity by the pockels cell and makes 11 round-trips through the cavity, amplifying up to $40 \mu \mathrm{J}$ before it is ejected by the pockels cell. A Faraday isolator separates the input and the output pulses. Another flashlamp pumped, Nd:glass rod amplifier is used in a two-pass configuration to provide an additional gain of 5. Afterwards, the beam is spatially filtered and then compressed in time using a pair of parallel diffraction gratings. The pulses are frequency doubled and quadrupled in a pair of BBO crystals. The UV pulse train is expanded and transport $15 \mathrm{~m}$ in vacuum to a final set of imaging optics which relay the beam to the photocathode.

\section{Temporal Pulse Shaping}

\section{Requirement and approach}

In order to produce electron beams with high charge and high brightness, the width of each laser pulse must be small compared with the RF period, but not so that the longitudinal space charge effects become unmanageable in the electron bunch, the normal design is 10ps. Besides that, the production of the flat beams needs the width of the laser pulse to be as long as $30 \mathrm{ps}$.

It is important for us to be able to vary the width of the laser pulse from a few picoseconds to tens of picoseconds in order to satisfy the requirements of different experiments. Since the grating compressor is set for a particular frequency chirp, we can change the width of the IR laser pulse by either varying the distance between two gratings or by changing the bandwidth of the laser pulse through the tuning of the oscillator cavity. Here, the grating compressor is set in such a way that the variation of the distance between two gratings will introduce extra phase shifts among the spectral components of the laser pulse, and the result of this is to change the laser pulse length. Also, the phase differences among the spectral components of the laser pulse are strongly dependent upon the bandwidth of the laser pulse, so does the laser pulse length after the grating compressor. Any variation in the bandwidth of the laser pulse will result in a different laser pulse length after the grating compressor while the setup of the grating compressor remains the same. 


\section{Experiment and result}

Two stages of second harmonic generation are used to convert the compressed infrared pulses at $\lambda=1054 \mathrm{~nm}$ to the fourth harmonic at $263.5 \mathrm{~nm}$. Since the second harmonic generation (SHG) is the degenerate case of nonlinear optical three wave mixing, the UV pulse length is directly correlated to the IR pulse length; we also observed this correlation in our experiment. Therefore, we can obtain the UV temporal pulse shaping by changing the IR pulse length.

A typical stepping autocorrelator is used to measure the autocorrelation of the compressed IR pulse after the grating compressor. A Hamamatsu C5680-21S streak camera with M5676 fast sweep module and a Pulnix progressive scan CCD camera measured the UV laser temporal profile. The schematic of IR autocorrelator is shown in Figure 2. Since the autocorrelation uses the leaked IR pulse from the reflection mirror after the grating compressor, we can simultaneously measure the IR pulse length by the autocorrelation and UV pulse length by the streak camera.

We did three experiments. First, we changed the distance between two gratings while keeping the bandwidth of the oscillator at a constant of $2.03 \mathrm{~nm}$. The IR pulse reached a maximal compression when the distance between two gratings was $789.75 \mathrm{~mm}$, as shown in Figure 3. Second, we changed the bandwidth of the oscillator while keeping the distance between two gratings at a constant of $789.75 \mathrm{~mm}$. We repeated the same experiment when the distance between two gratings was $842.25 \mathrm{~mm}$. The IR pulse length is strongly dependent upon the bandwidth of the oscillator, as shown in Figure 4. Finally, we simultaneously measured the IR pulse length by the autocorrelation and the UV pulse length by the streak camera. The result is shown in Figure 5. The ratio between the UV pulse length and the IR pulse length has two different values. The conversion from IR to UV is no longer intensity dependent when this process is saturated, and this situation corresponds to higher ratio between the UV and IR pulse length. There is a quadratic relationship between the intensity of the UV pulse and the intensity of the IR pulse when the conversion from IR to UV is not saturated, and this contributes to a factor of 0.5 smaller UV to IR length ratio compared to the case when the conversion is saturated. 


\section{Improvement in the Conversion Efficiency from IR to UV}

From the above experimental data, it is clear that the conversion from IR to UV is strongly dependent upon the IR photon density in the doubling crystal. Both the beam size and pulse length of the IR pulse in the crystal will influence the conversion efficiency. The experimental data shown in Figure 6 was taken by changing the intensity of the IR pulse through the rotation of the quarter-waveplate while keeping the IR pulse length at constant. Before the doubling crystal was saturated, the IR intensity was in the range of 0 to $0.17 \mathrm{~mJ}$, the conversion efficiency was increased when the IR intensity was increased. But after the IR intensity was increased to $0.17 \mathrm{~mJ}$, the conversion efficiency from IR to UV was slowly decreased.

\section{Application}

Different type of experiments requires different UV energy and pulse length. The conversion from IR to UV is strongly dependent upon the IR photon density in the doubling crystal. The beam size, pulse length and energy per pulse are the major parameters to determine the conversion from IR to UV, and they also can be changed during the experiment. In the situation when the high bunch charge is the most important parameter, the adjustment of the oscillator bandwidth can provide an efficient way to increase the energy per UV pulse. The strong correlation between the UV energy and the oscillator bandwidth is shown in Figure 7. In the situation when the bunch length is important, the oscillator bandwidth is pre-determined by a particular setup of the grating compressor, which is optimized for the bunch length requirement, and the only possible way to improve the conversion efficiency from IR to UV is to adjust the transverse size of the IR pulse in the crystal.

\section{References:}

[1] Alan R. Fry, "Novel Pulse Train Glass Laser for RF Photoinjectors" PhD thesis, University of Rochestor, 1996. 


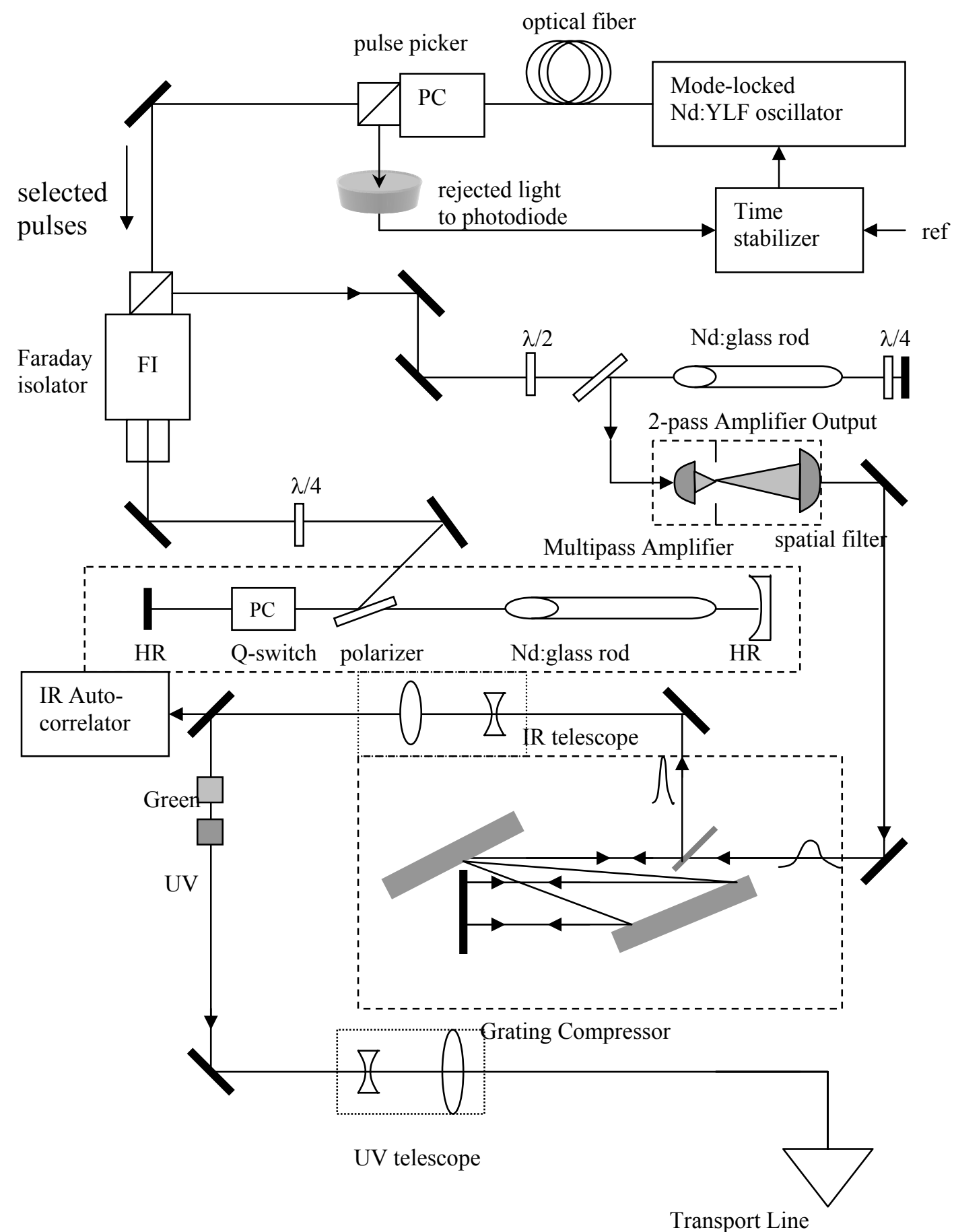

Figure 1 Schematic diagram of the laser system. 


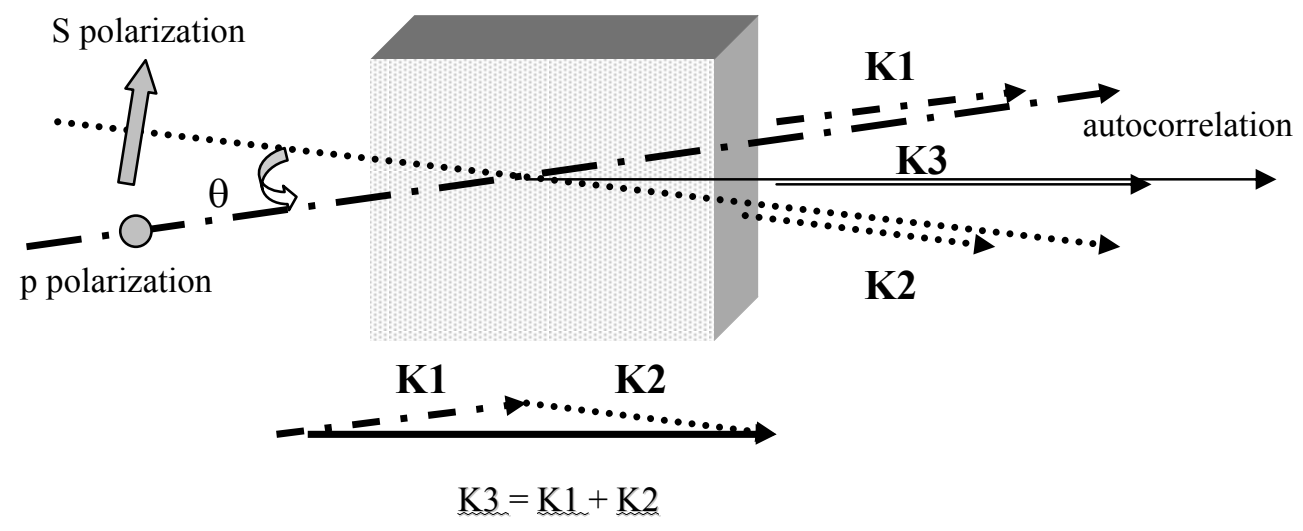

Figure 2 Schematic of IR autocorrelator. K1, K2, and K3 are wave numbers of the p-polarized IR pulse, s-polarized IR pulse, and the generated green pulse via the $2^{\text {nd }}$ harmonic. 


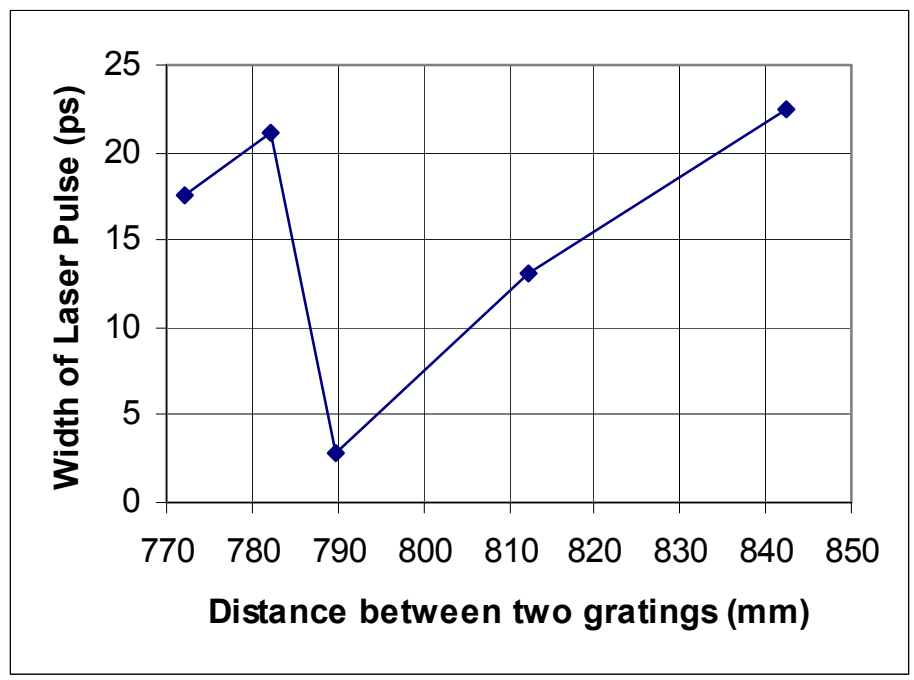

Figure 3 the IR pulse length $v$. the distance between two gratings. 


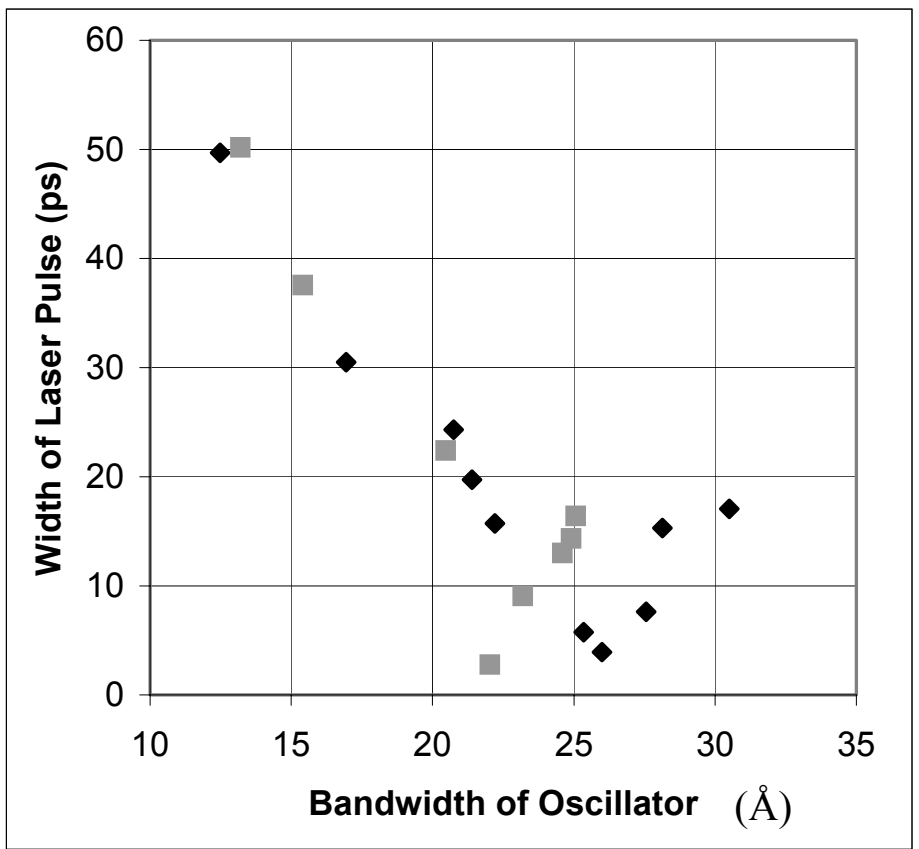

Figure 4 the IR pulse length $v s$. the bandwidth of the oscillator at two different distances between two gratings, $789.75 \mathrm{~mm}$ (grey) and $842.25 \mathrm{~mm}$ (blue). 

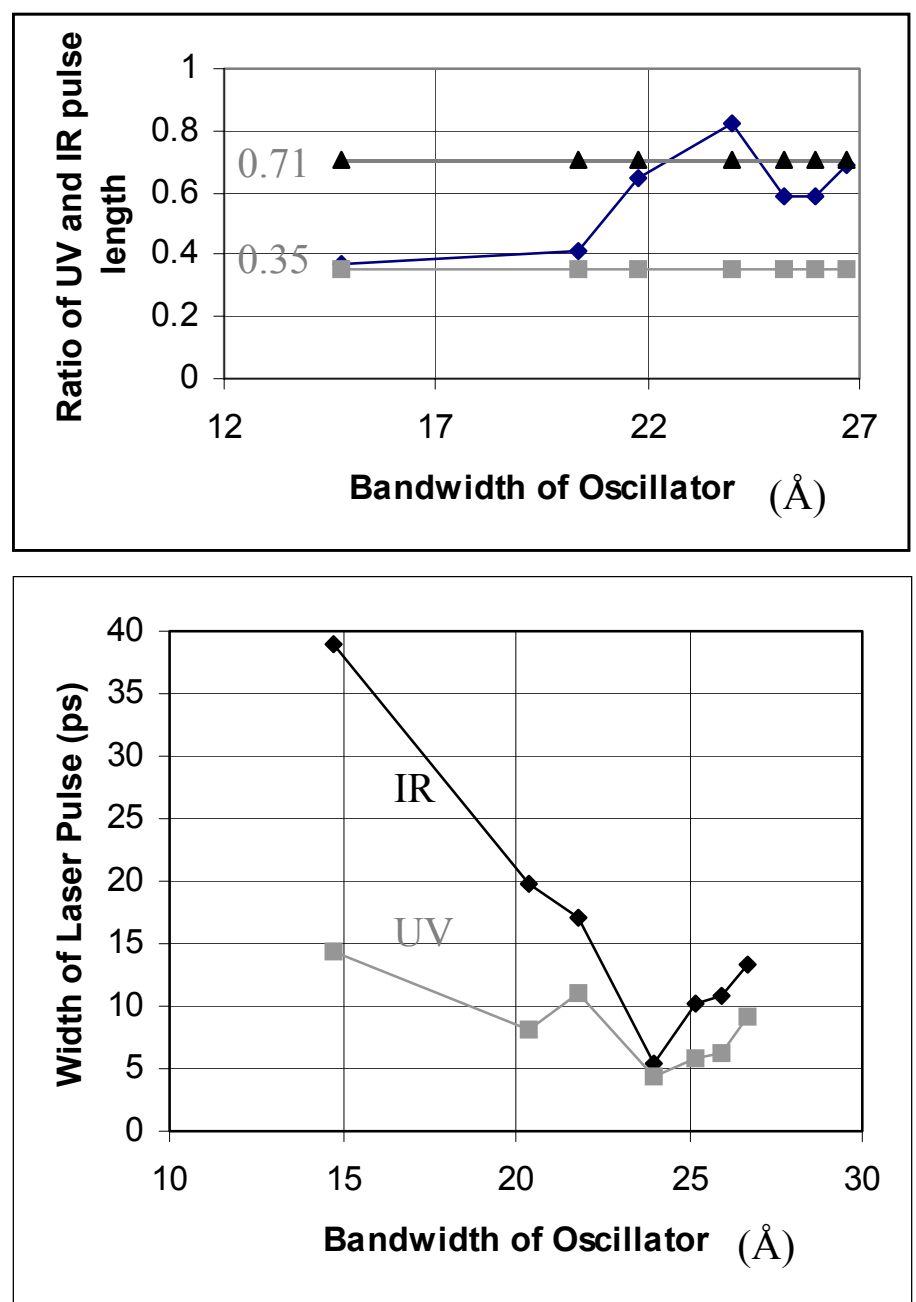

Figure 5 ratio of UV and IR pulse length $v s$. bandwidth of the oscillator (top), and the IR (blue) and UV (grey) pulse length vs. bandwidth of the oscillator. 

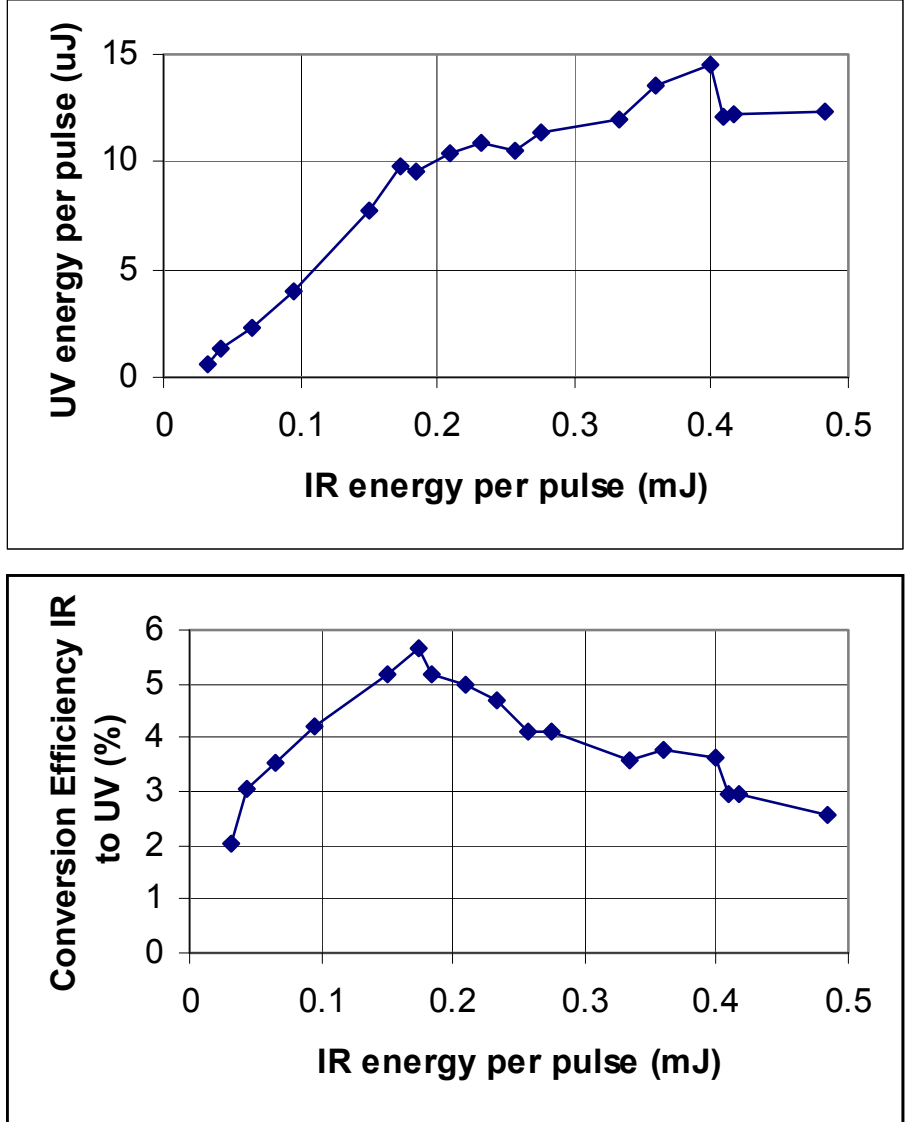

Figure 6 the UV energy per pulse $v s$. the IR energy per pulse (top), and the conversion efficiency of IR to UV vs. the IR energy per pulse (bottom). 


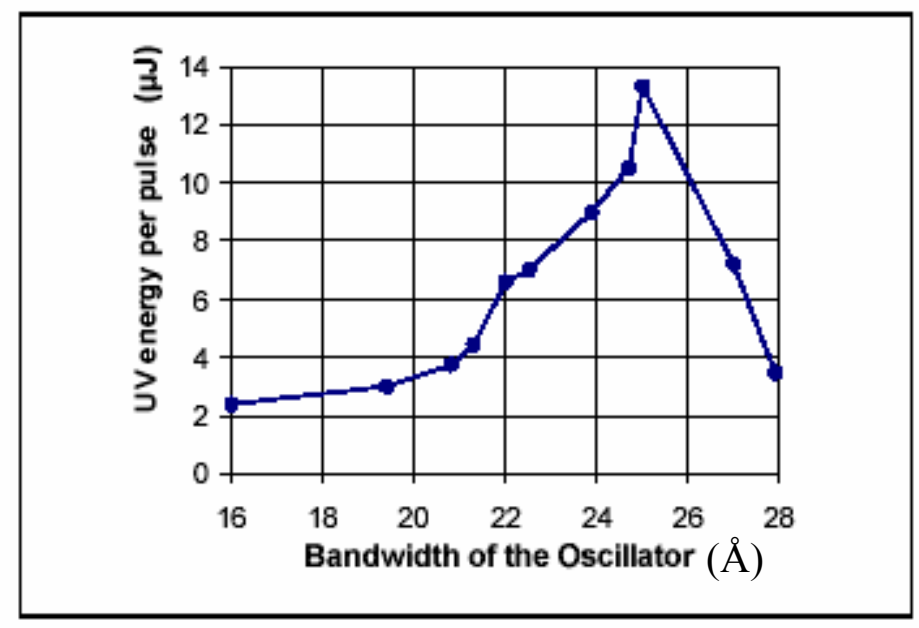

Figure 7 the UV energy per pulse $v s$. the bandwidth of the oscillator. 